\title{
Homotopy Lie Algebras of Gravity and their Braided Deformations
}

\section{Marija Dimitrijević Ćirić}

Faculty of Physics, University of Belgrade, Beograd, Serbia

E-mail: dmarija@ipb.ac.rs

\section{Grigorios Giotopoulos*}

Department of Mathematics, Heriot-Watt University, Edinburgh, United Kingdom Maxwell Institute for Mathematical Sciences, Edinburgh, United Kingdom

Email: gg $42 @$ hw.ac.uk

\section{Voja Radovanović}

Faculty of Physics, University of Belgrade, Beograd, Serbia

E-mail: rvoja@ipb.ac.rs

\section{Richard J. Szabo}

Department of Mathematics, Heriot-Watt University, Edinburgh, United Kingdom Maxwell Institute for Mathematical Sciences, Edinburgh, United Kingdom

Higgs Centre for Theoretical Physics, Edinburgh, United Kingdom

Email: R.J.Szabo@hw.ac.uk

\begin{abstract}
We describe the cyclic $L_{\infty}$-algebra formulation of classical general relativity without matter fields in the Einstein-Cartan-Palatini formalism. Using Drinfel'd twist deformation techniques, we define a noncommutative version of the theory with braided gauge symmetries. We introduce a notion of braided $L_{\infty}$-algebra, and use it to encode the symmetries, field content, field equations and Noether identities of noncommutative gravity.
\end{abstract}

Corfu Summer Institute 2019 "School and Workshops on Elementary Particle Physics and Gravity" (CORFU2019)

31 August - 25 September 2019

Corfù, Greece

Preprint: EMPG-20-10

${ }^{*}$ Speaker. 


\section{Introduction}

Recent advances in string theory suggest that the low-energy effective dynamics of closed strings in non-geometric flux compactifications may be described by noncommutative and even nonassociative deformations of gravity $[1,2,3,4,5]$. The framework of nonassociative differential geometry to accommodate such situations was developed in $[6,7,8,9]$. However, a suitable generalization of the Einstein-Hilbert action in the traditional metric formulation of general relativity has not yet been found. On the other hand, noncommutative and nonassociative deformations of gravity can be studied within the first order formulation of general relativity, which treats the theory as a deformation of a gauge theory and in which a deformed action can be constructed [10,11]. However, the deformed symmetries of these models do not behave as conventional gauge symmetries and have not been thorougly understood: an easy calculation shows that, with the naive definition of gauge transformations, nonassociativity obstructs closure of the gauge algebra.

The lack of closure of gauge transformations naturally suggests an approach based on $L_{\infty}$ algebras, which have a long history in both physics and mathematics. The main motivation behind this contribution, which is based on a series of papers [12,13], is to understand the symmetries, field equations and their Lagrangian formulations for noncommutative and nonassociative deformations of gravity via the formalism of $L_{\infty}$-algebras. $L_{\infty}$-algebras are generalizations of differential graded Lie algebras with infinitely-many graded antisymmetric brackets, related to each other by higher homotopy versions of the Jacobi identity. Hints of such structures may be traced back to higher spin gauge theories, where the closure of gauge transformations requires field-dependent gauge transformations [14]. They first appeared systematically in closed bosonic string field theory, where they govern the generalized gauge symmetries ${ }^{1}$ and dynamics of the theory [15], both necessarily involving infinitely-many higher brackets. In the mathematics literature, they are known to be dual to differential graded commutative algebras [16]. In [17] it was suggested that the complete data of any classical field theory with generalized gauge symmetries fit into truncated versions of cyclic $L_{\infty}$-algebras with finitely-many non-vanishing brackets, again encoding both gauge transformations and dynamics. The existence of such an $L_{\infty}$-algebra formulation is a consequence of the duality with the BV-BRST formalism for perturbative field theories, which is the differential graded commutative algebra incarnation [18].

Previous attempts at formulating general relativity in the $L_{\infty}$-algebra language have focused on the Einstein-Hilbert formulation. This necessitates perturbing the metric around a fixed background solution to the Einstein equations, which requires infinitely-many brackets to encode the complete dynamics [17]; similar approaches are also considered in [19, 20]. In contrast, the Einstein-CartanPalatini (ECP) formulation of gravity may be encoded in finitely-many brackets without requiring perturbative expansions. However, the standard approaches to noncommutative and nonassociative gauge theories typically also require infinitely-many brackets [21, 22], which is partly a consequence of the fact that the undeformed differential does not generally obey the usual Leibniz rule with respect to the deformed product. Furthermore, the twisted diffeomorphism symmetry of noncommutative gravity [23] does not fit nicely into an $L_{\infty}$-algebra framework involving only finitely-many brackets. This suggests finding a suitable deformation of the notion of an $L_{\infty}$-algebra

\footnotetext{
${ }^{1}$ By 'generalized' we mean gauge symmetries which are not restricted to automorphisms of principal bundles. Their action may not close off-shell, or they may also have a "non-linear" (field-dependent) action on the space of fields.
} 
to make it compatible with twisted symmetries and to accommodate the dynamics of noncommutative and nonassociative gauge theories. This leads to a mathematical object that we call a braided $L_{\infty}$-algebra whose definition will be sketched in the following; further details will appear in [13].

The purpose of the present contribution is to concisely summarise the contents of the papers $[12,13]$. We start by briefly recalling the definition of classical Einstein-Cartan-Palatini gravity, its symmetries and its Noether identities. We then recall the definition of cyclic $L_{\infty}$-algebras and their applications to perturbative field theories, and subsequently present the classical cyclic $L_{\infty}$-algebra formulation of ECP gravity which was developed and studied in detail in [12]. We proceed to describe a braided deformation of these $L_{\infty}$-algebras and the noncommutative theory of gravity that they determine, whose details will be developed in the forthcoming paper [13]. We explain how braided gauge symmetries induce Noether identities using the language of (braided) $L_{\infty}$-algebras, thus clarifying the physical content of braided symmetries. We conclude with a discussion of further questions and applications that may be explored using our formalism.

\section{General relativity in the Einstein-Cartan-Palatini formulation}

In this section we briefly review the dynamics and symmetries of classical general relativity in vacuum using the Einstein-Cartan-Palatini formalism. For ease of exposition, we restrict to the theory in dimension $d=3$, in Lorentzian signature, and without cosmological constant. Everything we say may be straightforwardly extended to higher dimensions $d \geq 4$ including a cosmological constant term, and for any signature of the metric; see [12] for further details.

\subsection{Field content and dynamics}

The Einstein-Cartan-Palatini formulation of gravity ${ }^{2}$ on a three-dimensional oriented manifold $M$ admitting a Lorentzian structure is defined by the action functional

$$
S_{\mathrm{ECP}}[e, \omega]=\int_{M} \operatorname{Tr}(e \curlywedge R)=\int_{M} \varepsilon_{a b c} e^{a} \wedge R^{b c} .
$$

The field content consists of an orientation-preserving bundle morphism $e: T M \rightarrow \mathscr{V}$ from the tangent bundle of $M$ to a fixed oriented vector bundle $\mathscr{V}$ isomorphic to $T M$ which is endowed with a fiberwise Minkowski metric $\eta$; this defines the coframe field $e \in \Omega^{1}(M, \mathscr{V})$, where $\mathscr{V}$ is sometimes called the 'fake tangent bundle'. The 2-form

$$
R=\mathrm{d} \omega+\frac{1}{2}[\omega, \omega] \in \Omega^{2}\left(M, P \times_{\mathrm{ad}} \mathfrak{s o}(1,2)\right)
$$

is the curvature of a connection $\omega$, called the spin connection, on the associated principal $\mathrm{SO}(1,2)$ bundle $P \rightarrow M$. The operation $\operatorname{Tr}: \Omega^{3}\left(M, \wedge^{3} \mathscr{V}\right) \rightarrow \Omega^{3}(M)$ is induced by the natural volume form of $\mathscr{V}$.

Since every orientable three-manifold is parallelizable, we can take $\mathscr{V}$ to be the trivial vector bundle $M \times \mathbb{R}^{1,2}$. Its associated SO $(1,2)$-bundle $P$ is then also trivial. In this case, the fields may be globally identified as 1 -forms $e \in \Omega^{1}\left(M, \mathbb{R}^{1,2}\right)$ and $\omega \in \Omega^{1}(M, \mathfrak{s o}(1,2))$. Using the isomorphism

\footnotetext{
${ }^{2}$ This is also known as the Einstein-Cartan-Sciama-Kibble theory, or simply the first order formalism for general relativity.
} 
$\mathfrak{s o}(1,2) \cong \wedge^{2} \mathbb{R}^{1,2}$, one identifies the spin connection as an element $\omega \in \Omega^{1}\left(M, \wedge^{2} \mathbb{R}^{1,2}\right)$, and then the $\curlywedge$-product separately takes the exterior products of the 'curved' spacetime differential forms on the manifold $M$ and 'flat' spacetime forms on the vector space $\mathbb{R}^{1,2}$. Then $\operatorname{Tr}: \wedge^{3} \mathbb{R}^{1,2} \rightarrow \mathbb{R}$ is simply the Hodge duality operator on Minkowski space $\left(\mathbb{R}^{1,2}, \eta\right)$.

The Euler-Lagrange derivatives which follow from varying the action functional (2.1) with respect to the coframe and spin connection respectively are

$$
F_{e}:=R \quad \text { and } \quad F_{\omega}:=T,
$$

where $T$ is the torsion 2-form which is defined as the covariant derivative of the coframe field:

$$
T:=\mathrm{d}^{\omega} e=\mathrm{d} e+\omega \wedge e \in \Omega^{2}\left(M, \mathbb{R}^{1,2}\right) .
$$

The vanishing of the second equation, $T=0$, implies that the torsion vanishes. When $e$ is a nondegenerate coframe field, or equivalently a bundle isomorphism, then this equation can be solved to identify the $\mathrm{SO}(1,2)$-connection $\omega$ with the Levi-Civita connection for the metric $g:=e^{*} \eta=$ $\eta^{a b} e_{a} \otimes e_{b}$. The vanishing of the first equation, $R=0$, is then the vacuum Einstein field equation in three dimensions, and thus the theory is classically equivalent to general relativity. Classical solutions correspond to flat spacetimes, which are locally isometric to Minkowski space and as such no gravitational waves exist in three dimensions. This is of course just the triviality of gravity in three spacetime dimensions. The only non-trivial features lie in the potential non-trivial topology of the spacetime.

The theory in arbitrary dimensionality $d \geq 3$ can be formulated by replacing the coframe field $e$ with the higher powers $e^{d-2}$ in the action functional (2.1), and in four dimensions the theory is again classically equivalent to general relativity which now possesses propagating degrees of freedom. However, in contrast to the Einstein-Hilbert formulation, the ECP theory makes sense for degenerate coframes $e$; this extension of general relativity to a theory with singularities is required for its $L_{\infty}$-algebra formulation, where the space of fields is required to be a vector or affine space.

\subsection{Gauge symmetries and Noether identities}

The (infinitesimal) gauge symmetries of the action functional (2.1) are given by the semi-direct product of the Lie algebras of vector fields on $M$ and of local Lorentz rotations:

$$
\Gamma(T M) \ltimes \Omega^{0}(M, \mathfrak{s o}(1,2)) .
$$

The diffeomorphism part is the standard symmetry of general relativity. The local rotation part may be interpreted as the freedom to change between orthonormal coframes for a given metric. The action of $(\xi, \rho) \in \Gamma(T M) \ltimes \Omega^{0}(M, \mathfrak{s o}(1,2))$ on the space of fields is given by

$$
\delta_{(\xi, \rho)}(e, \omega):=\left(\mathrm{L}_{\xi} e-\rho \cdot e, \mathrm{~L}_{\xi} \omega+\mathrm{d}^{\omega} \rho\right)
$$

where $\mathrm{L}_{\xi}$ is the Lie derivative along the vector field $\xi, \rho \cdot e$ denotes matrix multiplication, and $\mathrm{d}^{\omega} \rho=\mathrm{d} \rho+[\omega, \rho]$ is the covariant derivative.

By Noether's second theorem, gauge symmetries of an action functional are in bijection with (off-shell) differential identities between its Euler-Lagrange derivatives, called Noether identities. 
In the present case, these correspond to the pair of differential identities among the Euler-Lagrange derivatives $\left(F_{e}, F_{\omega}\right)$ given by

$$
\begin{aligned}
\mathrm{d} x^{\mu} \otimes \operatorname{Tr}\left(\iota_{\mu} \mathrm{d} e \curlywedge F_{e}+\imath_{\mu} \mathrm{d} \omega \curlywedge F_{\omega}-\imath_{\mu} e \curlywedge \mathrm{d} F_{e}-\imath_{\mu} \omega \curlywedge \mathrm{d} F_{\omega}\right) & =0 \in \Omega^{1}\left(M, \Omega^{3}(M)\right), \\
-F_{e} \wedge e+\mathrm{d}^{\omega} F_{\omega} & =0 \in \Omega^{3}\left(M, \mathbb{R}^{1,2}\right),
\end{aligned}
$$

where $\imath_{\mu}$ denotes the contraction with vectors $\partial_{\mu}=\frac{\partial}{\partial x^{\mu}}$ of the local holonomic frame dual to the basis $\left\{\mathrm{d} x^{\mu}\right\}$ of one-forms in a local coordinate chart on $M$, and we identify the vector space of 1-forms valued in 3-forms $\Omega^{1}\left(M, \Omega^{3}(M)\right)$ with $\Omega^{1}(M) \otimes \Omega^{3}(M)$. The first identity is the Noether identity corresponding to local diffeomorphism invariance $\delta_{(\xi, 0)} S_{\mathrm{ECP}}[e, \omega]=0$. The second identity gives the Noether identity corresponding to the local Lorentz gauge symmetry $\delta_{(0, \rho)} S_{\mathrm{ECP}}[e, \omega]=$ 0 , which also follows from the geometrical first Bianchi identity $\mathrm{d}^{\omega} T=R \wedge e$ by applying the covariant derivative to $F_{\omega}$. In three dimensions the first Bianchi identity coincides identically with the Noether identity for local Lorentz transformations, while in higher dimensions it only implies the Noether identity.

\section{3. $L_{\infty}$-algebras of classical gravity}

In this section we review the definition of cyclic $L_{\infty}$-algebras, and how classical field theories are formulated using them. We then present the $L_{\infty}$-algebras which organise ECP gravity in three dimensions.

\subsection{Cyclic $L_{\infty}$-algebras}

An $L_{\infty}$-algebra is a $\mathbb{Z}$-graded vector space $V=\bigoplus_{k \in \mathbb{Z}} V_{k}$ equipped with graded antisymmetric multilinear maps

$$
\ell_{n}: \otimes{ }^{n} V \longrightarrow V, \quad v_{1} \otimes \cdots \otimes v_{n} \longmapsto \ell_{n}\left(v_{1}, \ldots, v_{n}\right)
$$

for each $n \geq 1$, of degrees $\left|\ell_{n}\right|=2-n$, called $n$-brackets. The graded antisymmetry translates to

$$
\ell_{n}\left(\ldots, v, v^{\prime}, \ldots\right)=-(-1)^{|v|\left|v^{\prime}\right|} \ell_{n}\left(\ldots, v^{\prime}, v, \ldots\right)
$$

where we denote the degree of a homogeneous element $v \in V$ by $|v|$. Degreewise, the $n$-brackets are thus maps $\ell_{n}: V_{k_{1}} \otimes \cdots \otimes V_{k_{n}} \rightarrow V_{k_{1}+\cdots+k_{n}+2-n}$.

The $n$-brackets $\ell_{n}$ are required to fulfill infinitely-many identities, called homotopy relations, for each $n \geq 1$ :

$$
\sum_{i=1}^{n}(-1)^{i(n-i)} \sum_{\sigma \in \mathrm{Sh}_{i, n-i}} \chi\left(\sigma ; v_{1}, \ldots, v_{n}\right) \ell_{n+1-i}\left(\ell_{i}\left(v_{\sigma(1)}, \ldots, v_{\sigma(i)}\right), v_{\sigma(i+1)}, \ldots, v_{\sigma(n)}\right)=0,
$$

where, for each $i=1, \ldots, n$, the second sum runs over $(i, n-i)$-shuffled permutations $\sigma \in S_{n}$ of degree $n$ which are restricted as $\sigma(1)<\cdots<\sigma(i)$ and $\sigma(i+1)<\cdots<\sigma(n)$. The Koszul sign $\chi\left(\sigma ; v_{1}, \ldots, v_{n}\right)= \pm 1$ is determined from the grading by

$$
v_{\sigma(1)} \wedge \cdots \wedge v_{\sigma(n)}=\chi\left(\sigma ; v_{1}, \ldots, v_{n}\right) v_{1} \wedge \cdots \wedge v_{n}
$$


Let us examine the first three identities explicitly. The first relation for $n=1$ is

$$
\ell_{1}\left(\ell_{1}(v)\right)=0
$$

which states that the map $\ell_{1}: V \rightarrow V$ is a differential making $\left(V, \ell_{1}\right)$ into a cochain complex. The second relation with $n=2$ reads

$$
\ell_{1}\left(\ell_{2}\left(v_{1}, v_{2}\right)\right)=\ell_{2}\left(\ell_{1}\left(v_{1}\right), v_{2}\right)+(-1)^{\left|v_{1}\right|} \ell_{2}\left(v_{1}, \ell_{1}\left(v_{2}\right)\right),
$$

which states that $\ell_{1}$ is a graded derivation of the 2 -bracket $\ell_{2}$. The third relation for $n=3$ is

$$
\begin{aligned}
\ell_{2}\left(\ell_{2}\left(v_{1}, v_{2}\right), v_{3}\right)+(-1)^{\left(\left|v_{1}\right|+\left|v_{2}\right|\right)\left|v_{3}\right|} \ell_{2}\left(\ell_{2}\left(v_{3}, v_{1}\right), v_{2}\right)+(-1)^{\left(\left|v_{2}\right|+\left|v_{3}\right|\right)\left|v_{1}\right|} \ell_{2}\left(\ell_{2}\left(v_{2}, v_{3}\right), v_{1}\right) \\
=-\ell_{1}\left(\ell_{3}\left(v_{1}, v_{2}, v_{3}\right)\right) \\
\quad-\ell_{3}\left(\ell_{1}\left(v_{1}\right), v_{2}, v_{3}\right)-(-1)^{\left|v_{1}\right|} \ell_{3}\left(v_{1}, \ell_{1}\left(v_{2}\right), v_{3}\right)-(-1)^{\left|v_{1}\right|+\left|v_{2}\right|} \ell_{3}\left(v_{1}, v_{2}, \ell_{1}\left(v_{3}\right)\right),
\end{aligned}
$$

which states that the graded Jacobi identity for the 2-bracket $\ell_{2}$ holds generally only up to coherent homotopy. Thus $L_{\infty}$-algebras are (strong) homotopy deformations of differential graded Lie algebras which are the special cases where the ternary and all higher brackets vanish: $\ell_{n}=0$ for all $n \geq 3$. In general, the homotopy relations for $n \geq 3$ are generalized Jacobi identities.

A cyclic $L_{\infty}$-algebra is an $L_{\infty}$-algebra $V$ equipped with a non-degenerate graded symmetric bilinear pairing

$$
\langle-,-\rangle: V \times V \longrightarrow \mathbb{R}
$$

which satisfies the cyclicity property

$$
\left\langle v_{0}, \ell_{n}\left(v_{1}, v_{2}, \ldots, v_{n}\right)\right\rangle=-(-1)^{n+\left(\left|v_{0}\right|+\left|v_{n}\right|\right) n+\left|v_{n}\right| \sum_{i=0}^{n-1}\left|v_{i}\right|}\left\langle v_{n}, \ell_{n}\left(v_{0}, v_{1}, \ldots, v_{n-1}\right)\right\rangle .
$$

This is the natural generalization of the notion of an invariant inner product on a Lie algebra. Thus cyclic $L_{\infty}$-algebras generalize quadratic Lie algebras.

\section{2 $L_{\infty}$-algebra formulation of classical field theories}

Given a classical field theory defined by a (polynomial) action functional $S$ on a vector (or affine) space of dynamical fields with an irreducible set of generalized gauge symmetries, there is a 4-term $L_{\infty}$-algebra with underlying graded vector space given by

$$
V=V_{0} \oplus V_{1} \oplus V_{2} \oplus V_{3},
$$

where the different subspaces in degrees $0,1,2$ and 3 contain the gauge parameters, the dynamical fields, the Euler-Lagrange derivatives and the Noether identities, respectively. The linear parts of the gauge transformations, field equations and Noether identities are encoded by a differential $\ell_{1}: V \rightarrow V$, yielding a cochain complex $\left(V, \ell_{1}\right)$. This complex is further equipped with suitable higher brackets $\ell_{n}$ corresponding to the non-linear parts of the theory subject to the homotopy relations in order the recover the full symmetries and dynamics of the generalized gauge theory.

Given $\lambda \in V_{0}$ and $A \in V_{1}$, the gauge variations are encoded as the maps $A \mapsto A+\delta_{\lambda} A$ where

$$
\delta_{\lambda} A=\sum_{n=0}^{\infty} \frac{1}{n !}(-1)^{\frac{1}{2} n(n-1)} \ell_{n+1}(\lambda, A, \ldots, A),
$$


and the brackets involve $n$ insertions of the dynamical field $A$. The Euler-Lagrange derivatives are encoded as

$$
F_{A}=\sum_{n=1}^{\infty} \frac{1}{n !}(-1)^{\frac{1}{2} n(n-1)} \ell_{n}(A, \ldots, A),
$$

with the covariant gauge variations

$$
\delta_{\lambda} F_{A}=\sum_{n=0}^{\infty} \frac{1}{n !}(-1)^{\frac{1}{2} n(n-1)} \ell_{n+2}\left(\lambda, F_{A}, A, \ldots, A\right) .
$$

If the distribution in $T V_{1}$ spanned by the gauge parameters is involutive off-shell, that is, when $F_{A} \neq 0$, then the closure relation for the gauge algebra has the form

$$
\left[\delta_{\lambda_{1}}, \delta_{\lambda_{2}}\right] A=\delta_{\llbracket \lambda_{1}, \lambda_{2} \rrbracket_{A}} A,
$$

where

$$
\left[\lambda_{1}, \lambda_{2}\right]_{A}=-\sum_{n=0}^{\infty} \frac{1}{n !}(-1)^{\frac{1}{2} n(n-1)} \ell_{n+2}\left(\lambda_{1}, \lambda_{2}, A, \ldots, A\right) .
$$

The homotopy relations guarantee that the Jacobi identity is generally satisfied for any triple of maps $\delta_{\lambda_{1}}, \delta_{\lambda_{2}}$ and $\delta_{\lambda_{3}}$. Finally, the Noether identities are encoded by

$$
\sum_{n=0}^{\infty} \frac{1}{n !}(-1)^{\frac{1}{2} n(n-1)} \ell_{n+1}\left(F_{A}, A, \ldots, A\right)=0
$$

which vanishes identically (off-shell) as a consequence of the homotopy relations (3.3) with all entries set equal to $A$.

To encode the action functional of the gauge field theory, we assume that $V$ is equipped with a cyclic pairing of degree -3 , which makes $V$ into a cyclic $L_{\infty}$-algebra. In this case it is easy to see that the field equations $F_{A}=0$ follow from varying the action functional defined as

$$
S[A]:=\sum_{n=1}^{\infty} \frac{1}{(n+1) !}(-1)^{\frac{1}{2} n(n-1)}\left\langle A, \ell_{n}(A, \ldots, A)\right\rangle,
$$

since then cyclicity implies $\delta S[A]=\left\langle F_{A}, \delta A\right\rangle$. Gauge invariance of the action functional, $0=$ $\delta_{\lambda} S[A]=\left\langle F_{A}, \delta_{\lambda} A\right\rangle$, together with cyclicity on $V_{0}$ then imply the Noether identities (3.16).

\section{3 $L_{\infty}$-algebra picture of Einstein-Cartan-Palatini gravity}

We shall now present the $L_{\infty}$-algebra for ECP gravity in dimension $d=3$. The story may be extended to any $d \geq 3$, any signature, and incorporating a cosmological constant. For further details, along with explicit proofs of the homotopy relations and cyclicity conditions, we point the interested reader to [12].

The $L_{\infty}$-algebra which organises the gravity theory in three dimensions from Section 2 reduces to a differential graded Lie algebra, that is, the brackets $\ell_{n}$ vanish for all $n \geq 3$. The underlying cochain complex is given by

$$
V_{0} \stackrel{\ell_{1}}{\longrightarrow} V_{1} \stackrel{\ell_{1}}{\longrightarrow} V_{2} \stackrel{\ell_{1}}{\longrightarrow} V_{3}
$$


where

$$
\begin{aligned}
& V_{0}=\Gamma(T M) \times \Omega^{0}(M, \mathfrak{s o}(1,2)), \\
& V_{1}=\Omega^{1}\left(M, \mathbb{R}^{1,2}\right) \times \Omega^{1}(M, \mathfrak{s o}(1,2)), \\
& V_{2}=\Omega^{2}\left(M, \wedge^{2}\left(\mathbb{R}^{1,2}\right)\right) \times \Omega^{2}\left(M, \mathbb{R}^{1,2}\right), \\
& V_{3}=\Omega^{1}\left(M, \Omega^{3}(M)\right) \times \Omega^{3}\left(M, \mathbb{R}^{1,2}\right) .
\end{aligned}
$$

We denote elements of these vector spaces by $(\xi, \rho) \in V_{0},(e, \omega) \in V_{1},(E, \Omega) \in V_{2}$ and $(\mathscr{X}, \mathscr{P}) \in$ $V_{3}$. The differential is given by

$$
\ell_{1}(\xi, \rho)=(0, \mathrm{~d} \rho), \quad \ell_{1}(e, \omega)=(\mathrm{d} \omega, \mathrm{d} e) \quad \text { and } \quad \ell_{1}(E, \Omega)=(0, \mathrm{~d} \Omega) .
$$

The non-trivial 2-brackets are

$$
\begin{aligned}
& \ell_{2}\left(\left(\xi_{1}, \rho_{1}\right),\left(\xi_{2}, \rho_{2}\right)\right)=\left(\left[\xi_{1}, \xi_{2}\right],-\left[\rho_{1}, \rho_{2}\right]+\xi_{1}\left(\rho_{2}\right)-\xi_{2}\left(\rho_{1}\right)\right), \\
& \ell_{2}((\xi, \rho),(e, \omega))=\left(-\rho \cdot e+\mathrm{L}_{\xi} e,-[\rho, \omega]+\mathrm{L}_{\xi} \omega\right), \\
& \ell_{2}((\xi, \rho),(E, \Omega))=\left(-[\rho, E]+\mathrm{L}_{\xi} E,-\rho \cdot \Omega+\mathrm{L}_{\xi} \Omega\right), \\
& \ell_{2}((\xi, \rho),(\mathscr{X}, \mathscr{P}))=\left(\mathrm{d} x^{\mu} \otimes \operatorname{Tr}\left(\iota_{\mu} \mathrm{d} \rho \curlywedge \mathscr{P}\right)+\mathrm{L}_{\xi} \mathscr{X},-\rho \cdot \mathscr{P}+\mathrm{L}_{\xi} \mathscr{P}\right), \\
& \ell_{2}\left(\left(e_{1}, \omega_{1}\right),\left(e_{2}, \omega_{2}\right)\right)=-\left(\left[\omega_{1}, \omega_{2}\right], \omega_{1} \wedge e_{2}+\omega_{2} \wedge e_{1}\right), \\
& \ell_{2}((e, \omega),(E, \Omega))=\left(\mathrm{d} x^{\mu} \otimes \operatorname{Tr}\left(\iota_{\mu} \mathrm{d} e \curlywedge E+\iota_{\mu} \mathrm{d} \omega \curlywedge \Omega-\iota_{\mu} e \curlywedge \mathrm{d} E-\iota_{\mu} \omega \curlywedge \mathrm{d} \Omega\right),\right. \\
&E \wedge e-\omega \wedge \Omega) .
\end{aligned}
$$

As designed by (3.11)-(3.16), these brackets encode the symmetries and dynamics of threedimensional gravity. In summary:

- Gauge transformations of fields:

$$
\delta_{(\xi, \rho)}(e, \omega)=\left(-\rho \cdot e+\mathrm{L}_{\xi} e, \mathrm{~d} \rho-[\rho, \omega]+\mathrm{L}_{\xi} \omega\right)=\ell_{1}(\xi, \rho)+\ell_{2}((\xi, \rho),(e, \omega)) .
$$

- Euler-Lagrange derivatives:

$$
F_{(e, \omega)}=(R, T)=(\mathrm{d} \omega, \mathrm{d} e)+\frac{1}{2}([\omega, \omega], 2 \omega \wedge e)=\ell_{1}(e, \omega)-\frac{1}{2} \ell_{2}((e, \omega),(e, \omega)) .
$$

- Covariance of field equations:

$$
\delta_{(\xi, \rho)} F_{(e, \omega)}=\left(-[\rho, R]+\mathrm{L}_{\xi} R,-\rho \cdot T+\mathrm{L}_{\xi} T\right)=\ell_{2}\left((\xi, \rho), F_{(e, \omega)}\right) .
$$

- Noether identities:

$$
\begin{gathered}
\left(\mathrm{d} x^{\mu} \otimes \operatorname{Tr}\left(\iota_{\mu} e \curlywedge \mathrm{d} R-\imath_{\mu} \mathrm{d} e \curlywedge R+\imath_{\mu} \omega \curlywedge \mathrm{d} T-\imath_{\mu} \mathrm{d} \omega \curlywedge T\right), \mathrm{d}^{\omega} T-R \wedge e\right) \\
=\ell_{1}\left(F_{(e, \omega)}\right)-\ell_{2}\left((e, \omega), F_{(e, \omega)}\right) \\
=(0,0) .
\end{gathered}
$$


To write the action functional (2.1) in this language, we define a cyclic pairing of degree -3 by

$$
\langle(e, \omega),(E, \Omega)\rangle:=\int_{M} \operatorname{Tr}(e \curlywedge E+\Omega \curlywedge \omega)
$$

on $V_{1} \times V_{2}$. It is extended by

$$
\langle(\xi, \rho),(\mathscr{X}, \mathscr{P})\rangle:=\int_{M} \imath_{\xi} \mathscr{X}+\int_{M} \operatorname{Tr}(\rho \curlywedge \mathscr{P})
$$

on $V_{0} \times V_{3}$, where $l_{\xi}$ denotes contraction with the vector field $\xi$. Then the ECP action functional can be written as in (3.17) using the cyclic pairing (3.26):

$$
\begin{aligned}
S_{\mathrm{ECP}}[e, \omega] & =\int_{M} \operatorname{Tr}\left(e \curlywedge\left(\mathrm{d} \omega+\frac{1}{2}[\omega, \omega]\right)\right) \\
& =\frac{1}{2}\left\langle(e, \omega), \ell_{1}(e, \omega)\right\rangle-\frac{1}{6}\left\langle(e, \omega), \ell_{2}((e, \omega),(e, \omega))\right\rangle .
\end{aligned}
$$

One application of this formulation gives a new (and deeper) perspective on the well-known equivalence between three-dimensional gravity (with vanishing cosmological constant) and ChernSimons theory with gauge algebra $i s o(1,2)=\mathbb{R}^{3} \rtimes \mathfrak{s o}(1,2)$, the Lie algebra of the Poincare group in three dimensions [24]. The ECP theory in three dimensions possesses an extra "translation" symmetry, whose Noether identity is the second Bianchi identity $\mathrm{d}^{\omega} R=0$, and its $L_{\infty}$-algebra may be extended to include this new gauge symmetry. In the case of non-degenerate coframes (metrics) one can show that the gauge orbits under (infinitesimal) diffeomorphisms and "translations" coincide on-shell. However, if one allows for degenerate metrics then the "translation" gauge orbits strictly include the diffeomorphism orbits on-shell, thus rendering the diffeomorphisms as redundant symmetries. This may be used to show that the differential graded Lie algebra of ECP gravity including degenerate coframes is quasi-isomorphic (as an $L_{\infty}$-algebra) to the differential graded Lie algebra of Chern-Simons theory, thus extending the equivalence in an off-shell sense. See [12] for further details and applications.

In higher dimensions $d \geq 4$, the ECP $L_{\infty}$-algebra contains higher brackets and is no longer simply a differential graded Lie algebra, owing to the higher degree polynomial nature of the action functional (in the coframes) and the field equations. In [12] is shown that, for any spacetime dimension, these $L_{\infty}$-algebras describe the complete BV-BRST formulation of ECP gravity; in particular, in four dimensions our $L_{\infty}$-algebras are dual to the BV-BRST formulation of [25].

\section{Braided $L_{\infty}$-algebras of noncommutative gravity}

In this section we explain how suitable Drinfel'd twists give rise to a braided noncommutative deformation of the ECP gravity theory and its $L_{\infty}$-algebras. Again for brevity we state everything in three spacetime dimensions, which captures the main novelties of our braided gauge symmetry approach. The cases of higher spacetime dimensions, together with proofs of the claims which follow and further explanations, will appear in the forthcoming paper [13]. 


\subsection{Drinfel'd twist deformations of manifolds}

We recall how to twist a manifold to a noncommutative space using a Drinfel'd twist. Let $M$ be a manifold and consider its Lie algebra of diffeomorphisms $\Gamma(T M)$. The universal enveloping algebra $U \Gamma(T M)$ is the tensor algebra (over $\mathbb{R}$ ) of $\Gamma(T M)$, regarded as the free unital algebra generated by $\Gamma(T M)$, modulo the two-sided ideal generated by $\xi_{1} \xi_{2}-\xi_{2} \xi_{1}-\left[\xi_{1}, \xi_{2}\right]$ for all $\xi_{1}, \xi_{2} \in \Gamma(T M)$. It is naturally a cocommutative Hopf algebra with the coproduct $\Delta: U \Gamma(T M) \rightarrow U \Gamma(T M) \otimes U \Gamma(T M)$ given by

$$
\Delta(\xi)=\xi \otimes 1+1 \otimes \xi \quad \text { and } \quad \Delta(1)=1 \otimes 1
$$

for all $\xi \in \Gamma(T M)$, and extended as an algebra homomorphism to the whole of $U \Gamma(T M)$.

There is a symmetric monoidal category whose objects are $U \Gamma(T M)$-modules and whose morphisms are equivariant maps (see e.g. [26]). A $U \Gamma(T M)$-module algebra is an algebra object in this category, that is, an algebra $\mathscr{A}$ with a $U \Gamma(T M)$-action $\triangleright: U \Gamma(T M) \otimes \mathscr{A} \rightarrow \mathscr{A}$ which is compatible with the algebra multiplication via the coproduct $\Delta$. In the following the main example of interest will be the exterior algebra of differential forms $\mathscr{A}=\left(\Omega^{\bullet}(M), \wedge\right)$, which carries a $\Gamma(T M)$ action via the Lie derivative, extending to a $U \Gamma(T M)$-action via successive applications of the Lie derivative. This is a $U \Gamma(T M)$-module algebra since

$$
\xi \triangleright(\alpha \wedge \beta)=\mathrm{L}_{\xi}(\alpha \wedge \beta)=\mathrm{L}_{\xi} \alpha \wedge \beta+\alpha \wedge \mathrm{L}_{\xi} \beta=\wedge \circ \Delta(\xi) \triangleright(\alpha \otimes \beta)
$$

for all $\xi \in \Gamma(T M)$ and $\alpha, \beta \in \Omega^{\bullet}(M)$. In the following we will usually drop the symbol $\triangleright$ to simplify the notation.

A Drinfel'd twist is a (normalized) 2-cocycle of the Hopf algebra $U \Gamma(T M)$, which is specified by an invertible element $\mathscr{F} \in U \Gamma(T M) \otimes U \Gamma(T M)$. In most applications the twists are actually elements of formal power series expansions $\mathscr{F}=: \mathrm{f}^{k} \otimes \mathrm{f}_{k}$ in a deformation parameter, with all spaces concerned also similarly extended, but we do not indicate this in the notation for simplicity. For example, on $M=\mathbb{R}^{d}$ the standard example is the abelian Hermitean Moyal-Weyl twist

$$
\mathscr{F}=\exp \left(-\frac{\mathrm{i}}{2} \theta^{\mu v} \partial_{\mu} \otimes \partial_{v}\right),
$$

where $\left(\theta^{\mu v}\right)$ is a $d \times d$ antisymmetric real matrix. However, our considerations in the following apply to a more general class of Drinfel'd twists that we shall specify momentarily.

A Drinfel'd twist defines a new Hopf algebra structure on the universal enveloping algebra, which we denote by $U_{\mathscr{F}} \Gamma(T M)$. It has a twisted coproduct

$$
\Delta_{\mathscr{F}}(X):=\mathscr{F} \Delta(X) \mathscr{F}^{-1}
$$

for all $X \in U \Gamma(T M)$, where $\mathscr{F}^{-1}=: \overline{\mathrm{f}}^{k} \otimes \overline{\mathrm{f}}_{k}$. The deformation map defines a functorially equivalent symmetric monoidal category of $U_{\mathscr{F}} \Gamma(T M)$-modules. If $\mathscr{A}$ is a $U \Gamma(T M)$-module algebra, then we can deform the product on $\mathscr{A}$ by precomposing it with $\mathscr{F}^{-1}$. The cocycle condition on $\mathscr{F}$ guarantees that this defines an associative star-product, and it generally defines a noncommutative $U_{\mathscr{F}} \Gamma(T M)$-module algebra $\mathscr{A}_{\star}$, that is, an algebra object in the category of $U_{\mathscr{F}} \Gamma(T M)$-modules; if $\mathscr{A}$ is commutative, then $\mathscr{A}_{\star}$ is braided commutative. Let us spell this out explicitly on our main example of interest $\mathscr{A}=\left(\Omega^{\bullet}(M), \wedge\right)$. For $\alpha, \beta \in \Omega^{\bullet}(M)$ we set

$$
\alpha \wedge{ }_{\star} \beta:=\wedge \circ \mathscr{F}^{-1}(\alpha \otimes \beta)=\overline{\mathrm{f}}^{k} \alpha \wedge \overline{\mathrm{f}}_{k} \beta
$$


with graded commutativity controlled up to a braiding given by the triangular $\mathscr{R}$-matrix

$$
\mathscr{R}=\mathscr{F}_{21} \mathscr{F}^{-1}=: \mathrm{R}^{k} \otimes \mathrm{R}_{k}
$$

where $\mathscr{F}_{21}=\mathrm{f}_{k} \otimes \mathrm{f}^{k}$ is the twist with its legs flipped. Explicitly

$$
\alpha \wedge_{\star} \beta=(-1)^{|\alpha||\beta|} \overline{\mathrm{R}}^{k} \beta \wedge_{\star} \overline{\mathrm{R}}_{k} \alpha
$$

where $\mathscr{R}^{-1}=: \overline{\mathrm{R}}^{k} \otimes \overline{\mathrm{R}}_{k}$. The original action $\triangleright: U \Gamma(T M) \otimes \mathscr{A} \rightarrow \mathscr{A}$ is now compatible with the star-product via the twisted coproduct $\Delta_{\mathscr{F}}$ :

$$
X \triangleright\left(\alpha \wedge_{\star} \beta\right)=\wedge_{\star} \circ \Delta_{\mathscr{F}}(X)(\alpha \otimes \beta),
$$

and the twisted exterior algebra $\mathscr{A}_{\star}=\left(\Omega^{\bullet}(M), \wedge_{\star}\right)$ is now a $U_{\mathscr{F}} \Gamma(T M)$-module algebra. For further details on Drinfel'd twists see e.g. [27], and [28] for a review of their applications to twisted symmetries.

We will see below that $L_{\infty}$-algebras can be twisted in a similar manner, and thus twist the kinematics, symmetries and dynamics of classical field theories simultaneously in a consistent way. More generally, if $\mathscr{F}$ is a cochain twist (dropping the cocycle condition), then $U_{\mathscr{F}} \Gamma(T M)$ is a quasiHopf algebra and $\mathscr{A}_{\star}$ is a nonassociative algebra. This is the path towards a nonassociative theory of gravity taken in $[6,26,7,8,9,10,11]$, where further details of the cochain twist deformation may be found. However, while this is in principle a straightforward generalization, in the following we shall stick to the strictly associative noncommutative case for simplicity.

\subsection{Braided Einstein-Cartan-Palatini gravity}

Braided gauge symmetries and kinematics. One way to introduce a noncommutative theory of gravity is to start with the classical symmetries $\Gamma(T M) \ltimes \Omega^{0}(M, \mathfrak{s o}(1,2))$ and deform them consistently. That is, for any Drinfel'd twist $\mathscr{F}$, we define the braided Lie algebra structure given by the brackets

$$
\left[\rho_{1}, \rho_{2}\right]_{\star}:=[-,-] \circ \mathscr{F}^{-1}\left(\rho_{1} \otimes \rho_{2}\right)
$$

for $\rho_{1}, \rho_{2} \in \Omega^{0}(M, \mathfrak{s o}(1,2))$. The new bracket is now braided antisymmetric:

$$
\left[\rho_{1}, \rho_{2}\right]_{\star}=-\left[\overline{\mathrm{R}}^{k} \rho_{2}, \overline{\mathrm{R}}_{k} \rho_{1}\right]_{\star},
$$

and satisfies the braided Jacobi identity:

$$
\left[\rho_{1},\left[\rho_{2}, \rho_{3}\right]_{\star}\right]_{\star}=\left[\left[\rho_{1}, \rho_{2}\right]_{\star}, \rho_{3}\right]_{\star}+\left[\overline{\mathrm{R}}^{k} \rho_{2},\left[\overline{\mathrm{R}}_{k} \rho_{1}, \rho_{3}\right]_{\star}\right]_{\star},
$$

for all $\rho_{1}, \rho_{2}, \rho_{3} \in \Omega^{0}(M, \mathfrak{s o}(1,2))$. Similarly, one defines the braided Lie algebra of vector fields on $M$.

This differs from the usual approach to noncommutative gauge theory in the following way. In the standard approach one considers some matrix Lie algebra $\mathfrak{g}$, and deforms the associative matrix product of functions in the algebra $\Omega^{0}(M, \mathfrak{g})$. Then the star-bracket is defined as $\left[f^{\star}, g\right]:=$ $f \star g-g \star f$, which is subsequently required to close on $\Omega^{0}(M, \mathfrak{g})$ as an ordinary Lie algebra. This 
approach often requires an extension of the set of gauge symmetries, and it was first applied to gravity in [29,30]. This is the approach taken in [31] for ECP gravity, which necessarily introduces new degrees of freedom into the theory.

Instead, in our approach the braided bracket closes by definition on the same vector space, and hence avoids the introduction of new degrees of freedom. In terms of matrix multiplication, one can see that $\left[\rho_{1}, \rho_{2}\right]_{\star}=\rho_{1} \star \rho_{2}-\overline{\mathrm{R}}^{k} \rho_{2} \star \overline{\mathrm{R}}_{k} \rho_{1}$. Furthermore, the only coherent way of deforming $\Gamma(T M)$ simultaneously is as a braided gauge symmetry. Treating the two symmetries in a democratic way has positive outcomes. For example, the two braided Lie algebras may be combined in a single (braided) semi-direct product $\Gamma(T M) \ltimes_{\star} \Omega^{0}(M, \mathfrak{s o}(1,2))$, preserving the classical property of the gauge symmetries.

Furthermore, representations of the classical Lie algebras induce braided representations. In particular, we may define a notion of a braided coframe field $e \in \Omega^{1}\left(M, \mathbb{R}^{1,2}\right)$ and of a braided spin connection $\omega \in \Omega^{1}(M, \mathfrak{s o}(1,2))$ which transform as

$$
\delta_{\rho}^{\star} e=-\rho \star e \quad \text { and } \quad \delta_{\rho}^{\star} \omega=\mathrm{d} \rho-[\rho, \omega]_{\star}=\mathrm{d} \rho-\rho \star \omega+\overline{\mathrm{R}}^{k} \omega \star \overline{\mathrm{R}}_{k} \rho,
$$

where the products are defined by matrix multiplication with the star-product. These braided gauge transformations satisfy the braided Leibniz rule, for example

$$
\delta_{\rho}^{\star}(e \otimes \omega)=\delta_{\rho}^{\star} e \otimes \omega+\overline{\mathrm{R}}^{k} e \otimes \delta_{\overline{\mathrm{R}}_{k} \rho}^{\star} \omega .
$$

However, the exterior derivative $d$ itself acts via the ordinary, undeformed Leibniz rule. The braided torsion and braided curvature are naturally defined as

$$
T:=\mathrm{d} e+\omega \wedge_{\star} e \quad \text { and } \quad R:=\mathrm{d} \omega+\frac{1}{2}[\omega, \omega]_{\star},
$$

which as expected transform covariantly under (4.12) and (4.13).

The action of the braided diffeomorphisms is defined accordingly by twisting the classical action of vector fields. For example

$$
\delta_{\xi}^{\star} e=\mathrm{L}_{\xi}^{\star} e:=\mathrm{L}_{\overline{\mathrm{f}} k \xi}\left(\overline{\mathrm{f}}_{k} e\right)
$$

for $\xi \in \Gamma(T M)$.

Dynamics. The action functional for noncommutative ECP gravity in three dimensions is given by $[11,13]$

$$
S_{\mathrm{ECP}}^{\star}[e, \omega]=\int_{M} \operatorname{Tr}\left(e \curlywedge_{\star} R\right)=\int_{M} \varepsilon_{a b c} e^{a} \wedge_{\star} R^{b c} .
$$

This is the unique deformation of the classical action functional (2.1), given a twist $\mathscr{F}$ for which the deformed exterior product $\wedge_{\star}$ is (graded) cyclic commutative under the integral; for example, this is true for abelian twists, whereby $\mathscr{F}_{21}=\mathscr{F}^{-1}$. Such a graded cyclicity is also necessary for the variational principle to make sense. Together with the assumption that $\mathscr{F}$ is Hermitean, that is, it defines a Hermitean star-product $\wedge_{\star}$, then ensures that the action functional (4.16) is real-valued.

The action functional (4.16) is invariant under the action of braided gauge transformations of

$$
\Gamma(T M) \ltimes_{\star} \Omega^{0}(M, \mathfrak{s o}(1,2)) .
$$


The resulting field equations are captured by the vanishing of the Euler-Lagrange derivatives

$$
F_{e}^{\star}=R \quad \text { and } \quad F_{\omega}^{\star}=T-\frac{1}{2} \omega \wedge_{\star} e+\frac{1}{2} \overline{\mathrm{R}}^{k} \omega \wedge_{\star} \overline{\mathrm{R}}_{k} e .
$$

Thus the classical solutions may be interpreted as flat noncommutative spacetimes, however with torsion induced by the non-trivial braiding.

One distinctive feature of braided gauge symmetries is they do not produce new classical solutions: the field equations (4.18) are indeed braided covariant under (4.12), yet

$$
R[\omega]+\delta_{\rho}^{\star} R[\omega] \neq R\left[\omega+\delta_{\rho}^{\star} \omega\right],
$$

which is a consequence of the braided Leibniz rule (4.13). Another incarnation of this is seen in the braided gauge variations of the action functional (4.16):

$$
\delta_{\rho}^{\star} S_{\mathrm{ECP}}^{\star}[e, \omega] \neq \int_{M}\left(\delta_{\rho}^{\star} e \curlywedge_{\star} F_{e}^{\star}+\delta_{\rho}^{\star} \omega \curlywedge_{\star} F_{\omega}^{\star}\right),
$$

in contrast to field theories based on ordinary gauge symmetries. Hence the usual approach to Noether's second theorem also fails. However, a braided version of Noether's second theorem may be proved which again results in a set of a differential identities between the Euler-Lagrange derivatives $\left(F_{e}^{\star}, F_{\omega}^{\star}\right)$, that hold off-shell; as in the commutative case, these may be derived using the braided versions of the Bianchi identities [13]. This shows that the degrees of freedom are not independent, and justifies the interpretation of local braided symmetries as (generalized) 'gauge symmetries'. These identities are most elegantly phrased in terms of the corresponding braided $L_{\infty}$-algebra that we introduce below.

\subsection{Braided $L_{\infty}$-algebras}

Our definition of braided $L_{\infty}$-algebras is obtained from classical $L_{\infty}$-algebras. Let $\left(V,\left\{\ell_{n}\right\}\right)$ be an $L_{\infty}$-algebra object in the category of $U \Gamma(T M)$-modules. This means that the graded subspaces $V_{k}$ of $V$ are objects and the $n$-brackets $\ell_{n}$ define morphisms in this category, which are equivariant maps; concretely, this boils down to the action of $\Gamma(T M)$ on $V$ commuting with each of the $n$-brackets, via the trivial coproduct $\Delta$. A Drinfel'd twist $\mathscr{F}$ then induces an $L_{\infty}$-algebra object $\left(V,\left\{\ell_{n}^{\star}\right\}\right)$ in the category of $U_{\mathscr{F}} \Gamma(T M)$-modules, where $\ell_{1}^{\star}:=\ell_{1}$ and

$$
\ell_{n}^{\star}\left(v_{1} \otimes \cdots \otimes v_{n}\right):=\ell_{n}\left(v_{1} \otimes_{\star} \cdots \otimes_{\star} v_{n}\right)
$$

for $n \geq 2$, with $v \otimes_{\star} v^{\prime}:=\mathscr{F}^{-1}\left(v \otimes v^{\prime}\right)$ for $v, v^{\prime} \in V$. We call $\left(V,\left\{\ell_{n}^{\star}\right\}\right)$ a braided $L_{\infty}$-algebra, since its brackets are braided (graded) antisymmetric:

$$
\ell_{n}^{\star}\left(\ldots, v, v^{\prime}, \ldots\right)=-(-1)^{|v|\left|v^{\prime}\right|} \ell_{n}^{\star}\left(\ldots, \overline{\mathrm{R}}^{k} v^{\prime}, \overline{\mathrm{R}}_{k} v, \ldots\right),
$$

and satisfy the braided version of the homotopy relations (3.3). The first two relations for $n=1,2$ read as classically, while for $n=3$ the homotopy Jacobi identity (3.7) is modified to

$$
\begin{gathered}
\ell_{2}^{\star}\left(\ell_{2}^{\star}\left(v_{1}, v_{2}\right), v_{3}\right)-(-1)^{\left|v_{2}\right|\left|v_{3}\right|} \ell_{2}^{\star}\left(\ell_{2}^{\star}\left(v_{1}, \overline{\mathrm{R}}^{k} v_{3}\right), \overline{\mathrm{R}}_{k} v_{2}\right)+(-1)^{\left(\left|v_{2}\right|+\left|v_{3}\right|\right)\left|v_{1}\right|} \ell_{2}^{\star}\left(\ell_{2}^{\star}\left(\overline{\mathrm{R}}^{k} v_{2}, \overline{\mathrm{R}}^{l} v_{3}\right), \overline{\mathrm{R}}_{l} \overline{\mathrm{R}}_{k} v_{1}\right) \\
=-\ell_{1}^{\star}\left(\ell_{3}^{\star}\left(v_{1}, v_{2}, v_{3}\right)\right) \\
\quad-\ell_{3}^{\star}\left(\ell_{1}^{\star}\left(v_{1}\right), v_{2}, v_{3}\right)-(-1)^{\left|v_{1}\right|} \ell_{3}^{\star}\left(v_{1}, \ell_{1}^{\star}\left(v_{2}\right), v_{3}\right)-(-1)^{\left|v_{1}\right|+\left|v_{2}\right|} \ell_{3}^{\star}\left(v_{1}, v_{2}, \ell_{1}^{\star}\left(v_{3}\right)\right),
\end{gathered}
$$


and similarly for all generalized Jacobi identities with $n \geq 3$. The essential feature is that the permutation action in the identities (3.3) is enhanced by the application of the $\mathscr{R}$-matrix.

Similarly, cyclic pairings $\langle-,-\rangle: V \times V \rightarrow \mathbb{R}$ in the category of $U \Gamma(T M)$-modules induce braided cyclic pairings via

$$
\langle-,-\rangle_{\star}:=\langle-,-\rangle \circ \mathscr{F}^{-1} .
$$

However, for our choices of twists and pairings in field theory these become strictly cyclic.

Let us now write down the braided $L_{\infty}$-algebra of noncommutative gravity. For this, we first observe that the classical cyclic $L_{\infty}$-algebra for three-dimensional gravity from Section 3.3 is indeed an object in the category of $U \Gamma(T M)$-modules, and hence we obtain a cyclic braided $L_{\infty}$-algebra via twisting. The underlying cochain complex (3.18) is formally unchanged from the classical case. The resulting structure is a cyclic differential braided (graded) Lie algebra whose non-trivial 2-brackets are given by

$$
\begin{aligned}
\ell_{2}^{\star}\left(\left(\xi_{1}, \rho_{1}\right),\left(\xi_{2}, \rho_{2}\right)\right) & =\left(\left[\xi_{1}, \xi_{2}\right]_{\star},-\left[\rho_{1}, \rho_{2}\right]_{\star}+\mathrm{L}_{\xi_{1}}^{\star}\left(\rho_{2}\right)-\mathrm{L}_{\overline{\mathrm{R}}^{k} \xi_{2}}^{\star}\left(\overline{\mathrm{R}}_{k} \rho_{1}\right)\right), \\
\ell_{2}^{\star}((\xi, \rho),(e, \omega)) & =\left(-\rho \star e+\mathrm{L}_{\xi}^{\star} e,-[\rho, \omega]_{\star}+\mathrm{L}_{\xi}^{\star} \omega\right), \\
\ell_{2}^{\star}((\xi, \rho),(E, \Omega)) & =\left(-\rho \star E+\mathrm{L}_{\xi}^{\star} E,-\rho \star \Omega+\mathrm{L}_{\xi}^{\star} \Omega\right), \\
\ell_{2}^{\star}((\xi, \rho),(\mathscr{X}, \mathscr{P})) & =\left(\mathrm{d} x^{\mu} \otimes \operatorname{Tr}\left(\iota_{\mu} \mathrm{d}\left(\overline{\mathrm{f}}^{k} \rho\right) \curlywedge \overline{\mathrm{f}}_{k} \mathscr{P}\right)+\mathrm{L}_{\xi}^{\star} \mathscr{X},-\rho \star \mathscr{P}+\mathrm{L}_{\xi}^{\star} \mathscr{P}\right) \\
\ell_{2}^{\star}\left(\left(e_{1}, \omega_{1}\right),\left(e_{2}, \omega_{2}\right)\right) & =-\left(\left[\omega_{1}, \omega_{2}\right]_{\star}, \omega_{1} \wedge_{\star} e_{2}+\overline{\mathrm{R}}^{k} \omega_{2} \wedge_{\star} \overline{\mathrm{R}}_{k} e_{1}\right), \\
\ell_{2}^{\star}((e, \omega),(E, \Omega)) & =\left(\mathrm { d } x ^ { \mu } \otimes \operatorname { T r } \left(\imath_{\mu} \mathrm{d}\left(\overline{\mathrm{f}}^{k} e\right) \curlywedge \overline{\mathrm{f}}_{k} E+\iota_{\mu} \mathrm{d}\left(\overline{\mathrm{f}}^{k} \omega\right) \curlywedge \overline{\mathrm{f}}_{k} \Omega-\imath_{\mu}\left(\overline{\mathrm{f}}^{k} e\right) \curlywedge \mathrm{d}\left(\overline{\mathrm{f}}_{k} E\right)\right.\right. \\
& \left.\left.\quad-\iota_{\mu}\left(\overline{\mathrm{f}}^{k} \omega\right) \curlywedge \mathrm{d}\left(\overline{\mathrm{f}}_{k} \Omega\right)\right), \overline{\mathrm{R}}^{k} E \wedge_{\star} \overline{\mathrm{R}}_{k} e-\omega \wedge_{\star} \Omega\right) .
\end{aligned}
$$

We next show that this braided $L_{\infty}$-algebra organises all symmetries and dynamics of threedimensional noncommutative gravity. Firstly, one verifies that the action functional (4.16) is given as in the classical case:

$$
\begin{aligned}
S_{\mathrm{ECP}}^{\star}[e, \omega] & =\int_{M} \operatorname{Tr}\left(e \curlywedge_{\star}\left(\mathrm{d} \omega+\frac{1}{2}[\omega, \omega]_{\star}\right)\right) \\
& =\frac{1}{2}\left\langle(e, \omega), \ell_{1}^{\star}(e, \omega)\right\rangle_{\star}-\frac{1}{6}\left\langle(e, \omega), \ell_{2}^{\star}((e, \omega),(e, \omega))\right\rangle_{\star} .
\end{aligned}
$$

By cyclicity, the Euler-Lagrange derivatives follow from the same expansion as classically. The same expansions also hold for the expressions of the gauge transformations and the covariance of the field equations. However, braided gauge invariance of the action functional together with cyclicity result in the braided version of Noether's identities, due to the braided Leibniz rule. In summary:

- Braided gauge transformations of fields:

$$
\delta_{(\xi, \rho)}^{\star}(e, \omega)=\ell_{1}^{\star}(\xi, \rho)+\ell_{2}^{\star}((\xi, \rho),(e, \omega)) .
$$

- Euler-Lagrange derivatives:

$$
F_{(e, \omega)}^{\star}=\left(F_{e}^{\star}, F_{\omega}^{\star}\right)=\ell_{1}^{\star}(e, \omega)-\frac{1}{2} \ell_{2}^{\star}((e, \omega),(e, \omega)) .
$$


- Braided covariance of field equations:

$$
\delta_{(\xi, \rho)}^{\star} F_{(e, \omega)}^{*}=\left(-\left[\rho, F_{e}^{\star}\right]_{\star}+\mathrm{L}_{\xi}^{\star} F_{e}^{\star},-\rho \star F_{\omega}^{\star}+\mathrm{L}_{\xi}^{\star} F_{\omega}^{\star}\right)=\ell_{2}^{\star}\left((\xi, \rho), F_{(e, \omega)}^{\star}\right) .
$$

- Gauge redundancy via braided Noether identities:

$$
\begin{gathered}
\ell_{1}^{\star}\left(F_{(e, \omega)}^{\star}\right)-\frac{1}{2}\left(\ell_{2}^{\star}\left((e, \omega), F_{(e, \omega)}^{\star}\right)-\ell_{2}^{\star}\left(F_{(e, \omega)}^{\star},(e, \omega)\right)\right)+\frac{1}{4} \ell_{2}^{\star}\left(\overline{\mathrm{R}}^{k}(e, \omega), \ell_{2}^{\star}\left(\overline{\mathrm{R}}_{k}(e, \omega),(e, \omega)\right)\right) \\
=(0,0),
\end{gathered}
$$

where $\overline{\mathrm{R}}^{k}(e, \omega):=\left(\overline{\mathrm{R}}^{k} e, \overline{\mathrm{R}}^{k} \omega\right)$.

Note the compact form that the braided Noether identities take in this braided $L_{\infty}$-algebra formulation: Both identities corresponding to braided local Lorentz transformations and braided diffeomorphisms are included in (4.30) in a single line. In contrast, the explicit form of the identities becomes rather cumbersome when written out explicitly [13]. For example, the Noether identity corresponding to braided local Lorentz transformations is

$$
\begin{aligned}
\mathrm{d} F_{\omega}^{\star}-\frac{1}{2}\left(\overline{\mathrm{R}}^{k} F_{e}^{\star} \wedge_{\star} \overline{\mathrm{R}}_{k} e-\omega \wedge_{\star} F_{\omega}^{\star}+F_{e}^{\star} \wedge_{\star} e-\overline{\mathrm{R}}^{k} \omega \wedge_{\star} \overline{\mathrm{R}}_{k} F_{\omega}^{\star}\right) & \\
& +\frac{1}{4}\left(-\left[\omega, \overline{\mathrm{R}}^{k} \omega\right]_{\star} \wedge_{\star} \overline{\mathrm{R}}_{k} e+\overline{\mathrm{R}}^{k} \omega \wedge_{\star} \overline{\mathrm{R}}_{k} \omega \wedge_{\star} e+\overline{\mathrm{R}}^{k}\left(\omega \wedge_{\star} \omega\right) \wedge_{\star} \overline{\mathrm{R}}_{k} e\right)=0 .
\end{aligned}
$$

One may also verify the vanishing of the braided Noether identities identically (off-shell) by the braided homotopy identities.

This discussion extends to higher dimensions $d \geq 4$ in an analogous fashion, including now higher brackets of the braided $L_{\infty}$-algebra where appropriate [13].

\subsection{Outlook}

There are plenty of further natural questions and applications to explore from various physical perspectives. From the perspective of noncommutative gravity, the relation of our braided theory to the standard metric formulation of the theory [23], in which the torsion-free condition is assumed from the outset, can be explored. From the perspective of noncommutative field theories and braided $L_{\infty}$-algebras one should explore the necessary and sufficient conditions, both physical and mathematical, for the procedure we have discussed to succeed for other field theories. For example, it is straightforward to construct a braided noncommutative version of Yang-Mills theory [11] and of Chern-Simons theory [13]. Furthermore, it would be interesting to explore the possible dual incarnation of these structures as a "braided BV-BRST" formalism, and also the quantization of such braided gauge field theories in comparison to older work on braided quantum field theory [32, 33]. Lastly, our initial motivation — nonassociative gravity — may be now further advanced, which in this line of work will require yet a further generalization of our braided $L_{\infty}$-algebras using the formalism of cochain twist deformations and quasi-Hopf algebras.

From a mathematical perspective, the definition we presented of a braided $L_{\infty}$-algebra has an obvious generalization as an $L_{\infty}$-algebra object in any symmetric monoidal category. Given the vast and fruitful applications of $L_{\infty}$-algebras to various problems in mathematics, particularly in deformation theory, it would be interesting to pursue the applications of these braided generalizations. Specifically, they should play a role in operadic constructions where the role of the symmetric group is replaced with the braid group. 
Acknowledgments. We thank the organisors of the Corfu Summer Institute 2019 for the stimulating meeting and the opportunity to present the preliminary results of our work. The work of M.D.C. and V.R. is supported by Project ON171031 of the Serbian Ministry of Education, Science and Technological Development. The work of G.G. is supported by a Doctoral Training Grant from the UK Engineering and Physical Sciences Research Council. The work of R.J.S. was supported by the Consolidated Grant ST/P000363/1 from the UK Science and Technology Facilities Council.

\section{References}

[1] R. Blumenhagen and E. Plauschinn, "Nonassociative gravity in string theory?," J. Phys. A 44 (2011) 015401 [arXiv: 1010.1263 [hep-th]].

[2] D. Lüst, “T-duality and closed string noncommutative (doubled) geometry,” JHEP 1012 (2010) 084 [arXiv:1010.1361 [hep-th]].

[3] R. Blumenhagen, A. Deser, D. Lüst, E. Plauschinn and F. Rennecke, "Non-geometric fluxes, asymmetric strings and nonassociative geometry," J. Phys. A 44 (2011) 385401 [arXiv:1106.0316 [hep-th]].

[4] D. Mylonas, P. Schupp and R. J. Szabo, "Membrane sigma-models and quantization of non-geometric flux backgrounds," JHEP 1209 (2012) 012 [arXiv:1207.0926 [hep-th]].

[5] R. Blumenhagen, M. Fuchs, F. Hassler, D. Lüst and R. Sun, "Nonassociative deformations of geometry in double field theory," JHEP 1404 (2014) 141 [arXiv:1312.0719 [hep-th]].

[6] D. Mylonas, P. Schupp and R. J. Szabo, "Non-geometric fluxes, quasi-Hopf twist deformations and nonassociative quantum mechanics," J. Math. Phys. 55 (2014) 122301 [arXiv:1312.1621 [hep-th]].

[7] P. Aschieri and R. J. Szabo, "Triproducts, nonassociative star products and geometry of $R$-flux string compactifications," J. Phys. Conf. Ser. 634 (2015) 012004 [arXiv:1504.03915 [hep-th]].

[8] R. Blumenhagen and M. Fuchs, “Towards a theory of nonassociative gravity," JHEP 1607 (2016) 019 [arXiv:1604.03253 [hep-th]].

[9] P. Aschieri, M. Dimitrijević Ćirić and R. J. Szabo, "Nonassociative differential geometry and gravity with non-geometric fluxes," JHEP 1802 (2018) 036 [arXiv:1710.11467 [hep-th]].

[10] G. E. Barnes, A. Schenkel and R. J. Szabo, "Nonassociative geometry in quasi-Hopf representation categories II: Connections and curvature," J. Geom. Phys. 106 (2016) 234-255 [arXiv:1507.02792 [math.QA]].

[11] G. E. Barnes, A. Schenkel and R. J. Szabo, "Working with nonassociative geometry and field theory," Proc. Sci. 263 (2016) 081 [arXiv:1601.07353 [hep-th]].

[12] M. Dimitrijević Ćirić, G. Giotopoulos, V. Radovanović and R. J. Szabo, " $L_{\infty}$-algebras of Einstein-Cartan-Palatini gravity," arXiv:2003.06173 [hep-th].

[13] M. Dimitrijević Ćirić, G. Giotopoulos, V. Radovanović and R. J. Szabo, "Braided $L_{\infty}$-algebras and noncommutative gravity," in preparation.

[14] F. A. Berends, G. J. H. Burgers and H. van Dam, "On the theoretical problems in constructing interactions involving higher spin massless particles," Nucl. Phys. B 260 (1985) 295-322.

[15] B. Zwiebach, "Closed string field theory: Quantum action and the BV master equation," Nucl. Phys. B 390 (1993) 33-152 [arXiv:hep-th/9206084]. 
[16] T. Lada and J. Stasheff, "Introduction to sh Lie algebras for physicists,” Int. J. Theor. Phys. 32 (1993) 1087-1104 [arXiv:hep-th/9209099].

[17] O. Hohm and B. Zwiebach, " $L_{\infty}$-algebras and field theory," Fortsch. Phys. 65 (2017) 1700014 [arXiv:1701.08824 [hep-th]].

[18] B. Jurčo, L. Raspollini, C. Sämann and M. Wolf, " $L_{\infty}$-algebras of classical field theories and the Batalin-Vilkovisky formalism,” Fortsch. Phys. 67 (2019) 1900025 [arXiv:1809.09899 [hep-th]].

[19] M. Reiterer and E. Trubowitz, “The graded Lie algebra of general relativity,” arXiv:1812.11487 [math-ph].

[20] A. Nützi and M. Reiterer, "Scattering amplitudes in YM and GR as minimal model brackets and their recursive characterization," arXiv:1812.06454 [math-ph].

[21] R. Blumenhagen, I. Brunner, V. G. Kupriyanov and D. Lüst, "Bootstrapping noncommutative gauge theories from $L_{\infty}$-algebras,” JHEP 1805 (2018) 097 [arXiv:1803.00732 [hep-th]].

[22] V. G. Kupriyanov, "Noncommutative deformation of Chern-Simons theory,” Eur. Phys. J. C 80 (2020) 42 [arXiv:1905.08753 [hep-th]]

[23] P. Aschieri, C. Blohmann, M. Dimitrijević, F. Meyer, P. Schupp and J. Wess, "A gravity theory on noncommutative spaces”, Class. Quant. Grav. 22 (2005) 3511-3522 [arXiv:hep-th/0504183].

[24] E. Witten, “(2+1)-dimensional gravity as an exactly soluble system,” Nucl. Phys. B 311 (1988) 46-78.

[25] A. S. Cattaneo and M. Schiavina, "BV-BFV approach to general relativity: Palatini-Cartan-Holst action," arXiv:1707.06328 [math-ph].

[26] G. E. Barnes, A. Schenkel and R. J. Szabo, "Nonassociative geometry in quasi-Hopf representation categories I: Bimodules and their internal homomorphisms," J. Geom. Phys. 89 (2015) 111-152 [arXiv:1409.6331 [math.QA]].

[27] S. Majid, Foundations of Quantum Group Theory (Cambridge University Press, 1995).

[28] R. J. Szabo, “Symmetry, gravity and noncommutativity,” Class. Quant. Grav. 23 (2006) R199-R242 [arXiv:hep-th/0606233].

[29] A. H. Chamseddine, “Deforming Einstein’s gravity,” Phys. Lett. B 504 (2001) 33-37 [arXiv:hep-th/0009153].

[30] M. A. Cardella and D. Zanon, "Noncommutative deformation of four-dimensional Einstein gravity," Class. Quant. Grav. 20 (2003) L95-L104 [arXiv:hep-th/0212071].

[31] P. Aschieri and L. Castellani, "Noncommutative $D=4$ gravity coupled to fermions," JHEP 0906 (2009) 086 [arXiv:0902.3817 [hep-th]].

[32] R. Oeckl, "Braided quantum field theory,” Commun. Math. Phys 217 (2001) 451-473 [arXiv:hep-th/9906225].

[33] Y. Sasai and N. Sasakura, "Braided quantum field theories and their symmetries," Prog. Theor. Phys. 118 (2007) 785-814 [arXiv:0704.0822 [hep-th]]. 